\title{
Executive clock drawing correlates with performance-based functional status in people with combat-related mild traumatic brain injury and comorbid posttraumatic stress disorder
}

\author{
Brian W. Writer, DO; ${ }^{1 *}$ Jason E. Schillerstrom, MD $;^{2}$ Heather K. Regwan, DO $;^{2}$ Brent S. Harlan, MD $^{3}$ \\ ${ }^{1}$ Department of Psychiatry, San Antonio Military Medical Center, Lackland Air Force Base, TX; ${ }^{2}$ Department of Psy- \\ chiatry, School of Medicine, University of Texas Health Science Center at San Antonio, San Antonio, TX; ${ }^{3}$ Department \\ of Psychiatry, 1st Special Operations Medical Group, Hurlburt Field Air Force Base, FL
}

\begin{abstract}
Executive Clock Drawing Tasks (CLOX parts 1 and 2) can predict functional impairment. This study determined the correlation between CLOX and other psychometric screening instruments with the Structured Assessment of Independent Living Skills (SAILS)-defined performance-based functional status in people with combat-related mild traumatic brain injury (TBI) and comorbid posttraumatic stress disorder (PTSD). We hypothesized that CLOX would correlate significantly with functional performance. This prospective, crosssectional study design determined the correlation between a structured neuropsychological battery and functional status assessment. We calculated Pearson correlation coefficients between neuropsychological instruments and functional status scores. We entered neuropsychological measures correlating $p<$ 0.1 with functional status into a linear regression model to determine independent contributions. Fifteen Operation Iraqi Freedom veterans participated. Only CLOX1 correlated significantly with functional competency and efficiency. Only mean CLOX1 scores were significantly lower in those scoring below the median for SAILS competency and in those scoring above the median for SAILS efficiency. CLOX1 contributed significant variance to functional status independent of mood or anxiety symptoms and was not affected by age or time since injury. Executive dysfunction per the brief, easily administered CLOX1 is sensitive to functional status following combatrelated mild TBI, independent of PTSD anxiety with or without depression.
\end{abstract}

Key words: activities of daily living, cognitive disorder, concussion, executive functioning, functional status, head injury, mild traumatic brain injury, military combat injury, neuropsychological impairment, traumatic brain injury.

\section{INTRODUCTION}

More than 1.5 million servicemembers have been deployed to Iraq and Afghanistan since 2001, and of those returning stateside, at least 10 to 20 percent have experienced a traumatic brain injury (TBI) [1-2]. Reasons

\footnotetext{
Abbreviations: CLOX = Executive Clock Drawing Task, DOD $=$ Department of Defense, EXIT25 $=$ Executive Interview, GCS = Glasgow Coma Scale, HAM-D = Hamilton Depression Rating Scale, IRB = institutional review board, MIS $=$ Memory Impairment Screen, MMSE $=$ Mini-Mental Status Examination, $\mathrm{OEF}=$ Operation Enduring Freedom, OIF = Operation Iraqi Freedom, PCL $=$ Posttraumatic Stress Disorder Checklist, PTA $=$ posttraumatic amnesia, PTSD $=$ posttraumatic stress disorder, SAILS = Structured Assessment of Independent Living Skills, STVHCS $=$ South Texas Veterans Health Care System, TBI = traumatic brain injury, VA = Department of Veterans Affairs, WHMC = Wilford Hall Medical Center.

*Address all correspondence to Brian W. Writer, DO; Department of Psychiatry, 59 MHS/SGOWV1 2200 Bergquist Dr, Suite 1, Lackland Air Force Base, TX 78236; 210-2925941; fax: 210-292-5944. Email: brian.writer@us.af.mil DOI:10.1682/JRRD.2009.10.0162
} 
for the growing numbers of combat-related TBI are multifactorial, including increasingly large numbers of blast attacks, which are the most common etiology of TBI in the current war zones; improved body armor, resulting in improved survival rates; and better medical recognition of TBI [3]. Although TBI has been designated the "signature wound" of Operation Iraqi Freedom/Operation Enduring Freedom (OIF/OEF) [1], wound characteristics are quite diverse and classified according to (1) whether or not the skull has been breached (penetrating vs nonpenetrating) and (2) the severity of the initial impairment (mild, moderate, or severe) [4-8]. Severity is typically determined by the presenting Glasgow Coma Scale (GCS), the duration of loss and alteration of consciousness, and posttraumatic amnesia (PTA), as well as the presence or absence of structural imaging findings (Table 1) [4,6,810]. Although mild TBI accounts for the majority of cases [4,11], its criteria are an active area of debate [12], which has diagnostic, treatment, and prognostic implications.

TBI is associated with psychological, cognitive, and physical impairments [13-15]. Posttraumatic stress disorder (PTSD) is thought to be an especially relevant psychological comorbidity in combat veterans who have sustained a mild TBI given its high prevalence and additional morbidity load. PTSD point prevalence rates in OIF/OEF veterans who have sustained a mild TBI range from 27 percent [1] to 65 percent [16] and combat-related TBI reportedly doubles the risk for developing subsequent PTSD [17]. In addition to mediating health- and functional-related outcomes following a mild TBI [1], PTSD symptoms overlap with post-TBI sequelae such as persistent postconcussive symptoms [17] that are nonspecific [1]. This overlap in risk factors, occurrence, and clinical presentation has been challenging to distinguish for researchers and clinicians alike.
Although PTSD and other TBI-related sequelae presumably result in clinically significant functional impairments in OIF/OEF veterans, the diversity of trauma challenges our understanding of the interaction between PTSD and TBI, particularly in patients with mild TBI who may have impairments that go undetected by routine clinical examinations [12,18-19]. However, executive dysfunction may be a common pathway to functional impairment regardless of wound severity or psychological comorbidity.

Executive functioning can be conceptualized as higherorder cognitive processes that regulate complex behaviors through the integration of other cognitive domains [1820] such as attention and memory, which are frequently measured in the TBI literature, even in those with mild severity $[15,18,20]$. Executive dysfunction has been reported across the severity spectrum [11,19,21-24] with a reported point prevalence of 15 to 28 percent in those with mild TBI [25] and a 1-year prevalence of 25 to 36 percent in those with severe TBI [15]. A meta-analysis of civilian populations calculated a small effect size $(g=$ 0.3 ) for the adverse effect of mild TBI on executive functioning independent of time since injury [21]. Another civilian cross-sectional study reported that impaired executive functioning as demonstrated by a verbal fluency measure was the only mild TBI-related cognitive impairment to persist following multivariable analyses of premorbid intelligence, level of educational achievement, and self-reported depression [23]. Further contributing to the inherent challenge of comorbid PTSD following TBI is the available military literature, which has demonstrated that OIF/OEF veterans with comorbid PTSD following mild to moderate TBI events performed significantly worse on executive measures compared with those with only PTSD or TBI [24].

Functional impairments following TBI impede rehabilitation efforts, increase healthcare use, decrease quality

Table 1.

Traumatic brain injury severity stratification and classification.*

\begin{tabular}{lccc}
\hline \multicolumn{1}{c}{ Stratification } & Mild & Moderate & Severe \\
\hline Structural Imaging & Normal & Normal or abnormal & Normal or abnormal \\
LOC & $0-30$ min & $>30$ min and $<24 \mathrm{~h}$ & $>24 \mathrm{~h}$ \\
AOC (h) & Moment up to 24 & $>24^{\dagger}$ & $>24^{\dagger}$ \\
PTA (d) & $0-1$ & $>1$ and $<7$ & $3-8$ \\
GCS (score) & $13-15$ & $9-12$ & \\
Note: For purposes of injury stratification, GCS is measured $\geq 24 \mathrm{~h}$ and these stratification criteria do not apply to penetrating brain injuries where dura mater is breached. \\
${ }^{*}$ According to Department of Defense and Department of Veterans Affairs. \\
${ }^{\dagger}$ Severity based on other criteria. \\
AOC = alteration of consciousness, GCS = Glasgow Coma Scale, LOC = loss of consciousness, PTA = posttraumatic amnesia. \\
\hline \hline
\end{tabular}


of life, and increase risk of mortality [13,14,26]. It is assumed that neuropsychological functioning mediates functional decline [24], but little literature reports the relationship between these comorbidities, especially in TBI cohorts [11,13]. In fact, no studies describe the relationship between neuropsychological impairment using domain-specific cognitive instruments and functional status in people with combat-related TBI with or without additional PTSD anxiety. However, civilian literature, including cohort, cross-sectional, and review sources, suggests that executive function may be particularly relevant to functional outcome $[11,13,19,27-28]$. One prospective study of people with moderate to severe TBI constructed a latent "information processing speed" variable with an executive function task and demonstrated a moderate correlation between it and functional outcomes (standardized coefficient $t=0.68, p<0.05$ ) [28]. Moreover, they demonstrated that this construct meditated the relationship between injury severity and functional decline. A small cross-sectional study of subjects with mild TBI $(n=13)$ demonstrated that deficits in executive function correlated well with self-reported $(r=0.71, p<$ $0.01)$ and proxy-reported $(r=0.6, p<0.03)$ functional participation scores [11]. Identifying cognitive screening instruments that predict functional status and can be used in multiple settings, including the combat theater, should be a priority for TBI healthcare providers. Screening instruments sensitive to executive function may be particularly sensitive to functional abilities even in those with mild TBI.

Clock drawing tasks have emerged as a convenient method for assessing executive function. However, not all clock drawing tasks are necessarily "executive" and some, such as the Executive Clock Drawing Task (CLOX), correlate better with other putative executive measures such as the Executive Interview (EXIT25) [2930]. CLOX is divided into two parts, CLOX1 and CLOX2. CLOX1 is a command-directed clock drawing task that correlates significantly but moderately with other putative executive measures. CLOX2 is a clock copying task sensitive to visuospatial ability and constructional praxis. Poor CLOX1 performance is associated with functional impairments in elderly residing in continuing-care retirement communities, elderly transitioning to 24-hour care, and increased mortality [29,31]. Given its strong association with functional decline in longitudinal studies, we would expect that it would correlate well with cross-sectional performance-based func- tional assessments in people with TBI who are prone to frontal systems injury.

The purpose of this pilot study was to determine the correlation between executive clock drawing and performance-based functional status in people with combatrelated mild TBI and comorbid PTSD anxiety. Cohorts of military servicemembers with TBI differ from civilian cohorts at time of injury with respect to use of alcohol and other substances, employment status, mechanism of injury (blast injuries), and the PTSD-potentiating war environment [3]. Therefore, we cannot assume that predictors for postinjury functional status are necessarily the same between these two groups. However, in light of a lack of available military data, we are left to formulate our hypothesis a priori based on existing civilian findings that executive dysfunction has been associated with impaired functional status. As such, we hypothesized that CLOX1 would correlate significantly with functional performance and that subjects with better functional status scores would have higher mean clock drawing performance. Considering the typically brief natural recovery time (e.g., hours to days) following mild TBI $[4,12]$, as well as evidence that injury and/or subject characteristics such as comorbid psychological sequelae (e.g., PTSD anxiety and/or depression) can contribute to worsened neuropsychological [24] and/or functional status [1], we also examined our data for significant correlations between these characteristics and psychometric performance and functional status in an effort to mitigate potential confounding of results.

\section{METHODS}

\section{Participants}

From November 2007 through April 2009, subjects with combat-related TBI were referred from the Defense and Veterans Brain Injury Center located at Wilford Hall Medical Center (WHMC), Lackland Air Force Base, Texas, or from the Physical Medicine and Rehabilitation Department located at the South Texas Veterans Health Care System (STVHCS), San Antonio, Texas.

To be included in the study, subjects had to be Active Duty, separated, or retired veterans from any military branch; have a medically documented combat-related mild TBI as part of their standard of care evaluation according to Department of Defense (DOD) and Department of Veterans Affairs (VA) guidelines [4]; and have a 
diagnosis of PTSD documented in the medical record. Essentially, we restricted participation in this study to those with combat-related mild TBI and comorbid PTSD. Subjects had to be able to physically participate in neuropsychological and functional status assessments and be $\geq 17$ years old. The referral sources diagnosed the occurrence of a TBI using a standardized clinical interview of subjects performed by trained medical personnel who took into consideration all available supporting data including, but not limited to, medical records (including in-theatre records) and collateral information. We considered any diagnosed TBI that occurred during an OIF or OEF deployment a combat-related TBI. The referral sources then used identical injury stratification criteria to determine the severity of the diagnosed TBI, which ranged from mild to severe. We based the stratification criteria on DOD and VA guidelines [4], which consider multiple pieces of clinical data at the time of TBI, including brain structural imaging, loss and alteration of consciousness, PTA, and the GCS (Table 1).

We excluded participants if they had sensory or language limitations precluding neuropsychological testing, if they had non-combat-related TBI and/or a moderate or severe TBI, or if they were so cognitively impaired that they could not provide meaningful consent.

\section{Study Design}

Prior to subject enrollment, we trained each physician to perform the neuropsychological and functional assessment battery to ensure reliability in test administration and scoring. We collected demographic, medical, and psychiatric history, including a review of existing medical records for current (and/or pre-TBI) psychiatric diagnoses such as PTSD. For clarity, we determined psychiatric diagnoses by subject disclosure with concurrent review of existing medical records for clarification. A psychiatrist then administered the following standardized 60- to 90- minute neuropsychological and functional assessment battery:

1. CLOX: The CLOX is divided into two parts. For CLOX1, we asked subjects to construct a clock at the command, "Draw me a clock that says 1:45. Set the hands and numbers on the face so that a child could easily read them.” The instructions could be repeated until they were clearly understood, but we allowed no further assistance once the subject began to draw. For CLOX2, which assesses visuospatial ability, we asked subjects to copy a clock drawn by the examiner. We scored their performance on each clock from 0 to 15 according to standardized scoring directions (based on 15 categories all worth one point each), with higher scores representing better performance. Inter-rater reliability (CLOX1: $r=0.94$, CLOX2: $r=0.93$; both $p<$ 0.001 ) and internal consistency (Cronbach $\alpha=0.82$ ) were high for both tasks [29].

2. Mini-Mental Status Examination (MMSE): The MMSE is a measure of global cognition, scored from 0 to 30 with higher numbers representing better performance [32].

3. EXIT25: The EXIT25 is a 25-item assessment of executive function, scored from 0 to 50 with higher numbers representing worse performance [33].

4. Memory Impairment Screen (MIS): The MIS is a measure of delayed free- and cued-recall memory, scored from 0 to 8 with higher scores representing better performance [34].

5. Posttraumatic Stress Disorder Checklist (PCL): The PCL is a 17-item self-reported screening measure for military-related PTSD, each item rated from 1 to 5 with higher scores representing increased severity [35].

6. Hamilton Depression Rating Scale (HAM-D): The HAM-D is a 17-item scale that assesses depression, scored from 0 to 34 with scores $>8$ generally considered significant for depression [36].

7. Structured Assessment of Independent Living Skills (SAILS): The SAILS assesses instrumental activities of daily living and measures both competency and efficiency (time to completion). This performance-based instrument simulates and measures money-related skills (counting money, buying objects, making change), bill paying and check writing, telephone use, medication use, and calendar and clock skills [37]. For example, we evaluated and timed the subject on their ability to fill out a check for a pretend utility statement. We modified the scoring of the instrument, which was scaled such that lower scores and longer time to completion represented worse performance.

\section{Data Analysis}

We used Systat 11 (Systat Software, Inc; Chicago, Illinois) for statistical analyses. We compared mean demographic and neuropsychological performance scores between subjects above versus below the median for SAILS competency and efficiency using the Student $t$-test. We calculated Pearson correlation coefficients between age, time since injury, each neuropsychological instrument, and functional status score. We used both SAILS 
competency and efficiency as dependent outcome measures. Resulting significant correlations were Bonferroni, adjusted $(p<0.003)$ to mitigate chance significant correlations. We entered psychometric instruments with univariable correlations $(p<0.10)$ into a linear regression model to determine independent contributions to SAILS competency and efficiency.

\section{RESULTS}

Fifteen male OIF veterans consented to participate. The sample consisted of 60 percent Army, 27 percent Air Force, and 13 percent Marine Corps. For the sample, age was $32.9 \pm 8.6$ years (mean \pm standard deviation [SD] unless otherwise specified). Of the total sample, 87 percent (13 of 15 subjects) had no psychiatric diagnosis before the mild TBI. A depression diagnosis was documented in 40 percent of the sample. Of the sample, 67 percent used selective serotonin reuptake inhibitors. Only one subject was prescribed an antiepileptic medication. Time since injury was $29.1 \pm 16.1$ months (range: 6-60 months) (Table 2).

CLOX1 was the only neuropsychological measure that correlated significantly with SAILS competency $(r=$ $0.79 ; p<0.001)$ and efficiency $(r=-0.81 ; p<0.001)$ (Figure). Prior to Bonferroni correction, SAILS efficiency correlated with PCL $(r=0.57, p=0.03)$ and approached significance with HAM-D $(r=0.46, p=$ $0.08)$. CLOX1 correlated modestly with both PCL ( $r=$ $-0.66, p=0.007)$ and HAM-D $(r=-0.56, p=0.03)$. Nei- ther age nor time since injury correlated with SAILS competency, SAILS efficiency, or CLOX1.

We constructed two linear regression models to determine if CLOX1 performance contributed variance to SAILS efficiency independent of anxiety or depression. CLOX1 contributed independent variance to SAILS efficiency when covaried for PCL $\left(R^{2}=0.66, p=0.005\right)$ and HAM-D $\left(R^{2}=0.66, p=0.002\right)$, which were both rendered insignificant. As no other neuropsychological measure approached univariate correlation at $p<0.1$ with SAILS efficiency, we did not enter them into our linear regression model. Furthermore, as no neuropsychological measures except for CLOX1 significantly correlated with SAILS competency, we did not subject SAILS competency to multivariable analysis.

The median SAILS competency score was 55. Mean CLOX1 scores in subjects scoring below the SAILS performance median $(n=7)$ were significantly lower than scores for those scoring at or above the median $(n=$ 8 ) and crossed the published threshold for a failing score representing the lower fifth percentile for young adults $(9.4 \pm 4.1$ vs $13.3 \pm 0.5, t=-2.6, p=0.02)$ [29]. The median SAILS efficiency time was 5.1 minutes. Mean CLOX1 scores in subjects scoring above the median $(n=$ 7) were significantly lower than for those scoring at or below the median $(n=8)$ and again crossed the published cutoff point for clinically significant impairment (9.6 \pm 4.3 vs $13.1 \pm 0.6, t=2.3, p=0.04$ ). No significant mean differences were found for age or any other psychometric measure for either SAILS competency or efficiency.

Table 2.

Cognitive, mood, and functional performance in people with combat-related traumatic brain injury $(n=15)$.

\begin{tabular}{lcc}
\hline \multicolumn{1}{c}{ Assessment } & Range & Mean \pm SD \\
\hline Cognitive Assessment (score) & & $11.5 \pm 3.4$ \\
CLOX1 & $2-14$ & $13.7 \pm 1.3$ \\
CLOX2 & $10-15$ & $8.7 \pm 3.9$ \\
EXIT25 & $4-18$ & $5.9 \pm 1.6$ \\
MIS & $4-8$ & $28.3 \pm 1.3$ \\
MMSE & $26-30$ & $57.3 \pm 18.6$ \\
Mood Assessment (score) & $27-88$ & $18.0 \pm 10.3$ \\
$\quad$ PLC & $4-36$ & $54.5 \pm 2.4$ \\
HAM-D & $49-57$ & $5.4 \pm 2.2$ \\
Functional Assessment & $2-10$ & \\
$\quad$ SAILS Competency (score) & \\
$\quad$ SAILS Efficiency (min) & \\
\hline CLOX1 = Executive Clock Drawing Task part 1, CLOX2 = Executive Clock Drawing Task part 2, EXIT25 = Executive Interview, HAM-D = Hamilton Depression \\
Rating Scale, MIS = Memory Impairment Screen, MMSE = Mini-Mental Status Examination, PCL = Posttraumatic Stress Disorder Checklist, SAILS = Structured \\
Assessment of Independent Living Skills, SD = standard deviation. \\
\hline \hline
\end{tabular}



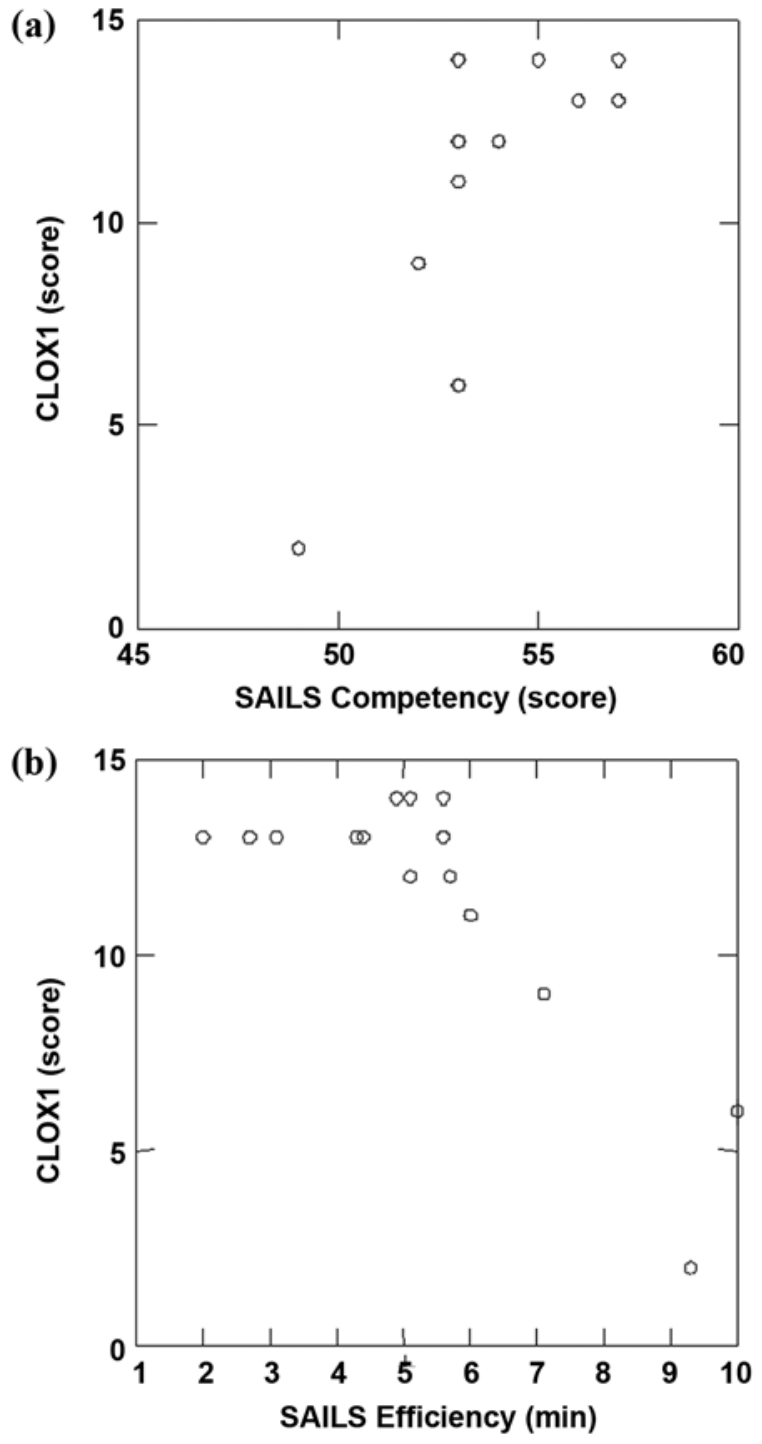

Figure.

Scatter-plot matrices for Executive Clock Drawing Task part 1 (CLOX1) performance versus (a) Structured Assessment of Independent Living Skills (SAILS) competency (Pearson $r=0.79, p<0.001$ ) and (b) SAILS efficiency (Pearson $r=-0.81, p<0.001$ ).

\section{DISCUSSION}

Our findings suggest that executive functioning as measured by CLOX1 may be particularly sensitive to functional status in servicemembers with comorbid PTSD following a combat-related mild TBI. Because executive function is a multidimensional process and the cortical, subcortical, and white matter regions governing it occupy 40 percent of brain volume, no single putative executive measure will be sensitive to all impairments. In this sample population, CLOX1-defined executive functioning correlated well with SAILS-defined functional competency (performance ability and accuracy) and efficiency (performance speed). Furthermore, CLOX1 performance contributed variance independent of comorbid PTSD anxiety symptom burden with or without additional depression or other potentially confounding subject and injury characteristics. Similarly, worse comorbid PTSD anxiety correlated with and worse depression trended toward worse SAILS efficiency, which is not surprising given the psychological trauma endured by Global War on Terrorism veterans. However, neither PTSD anxiety nor depression ultimately contributed independent variance. Nevertheless, comorbid psychological symptoms (especially PTSD anxiety) may contribute to aspects of persistent functional impairment following combat-related mild TBI.

These pilot findings should be approached with caution given important study limitations. First, we did not have a matched noncombat TBI comparison group, making it impossible to distinguish the TBI-related effects from the combat-related effects on neuropsychological and functional performance. This is an especially relevant potential limitation considering that our post hoc sample consisted entirely of people with mild TBI and comorbid PTSD as evidenced by available medical records and as supported by the mean PCL score (57.3), which approached suggested Keen cutoff point criteria for a PTSD diagnosis [38]. Although we used mood and anxiety as covariates in our linear regression models to determine the independent association between CLOX1defined executive function and functional status, we recognize that our pilot findings should be approached critically in light of recent literature that has highlighted the mediating effects of PTSD and mood on health-related functional outcomes in OIF/OEF combat veterans with mild TBI [1,16,21]. However, it should also be noted that a dichotomous conceptualization of the relative effect of TBI-related effects on functional status (brain-behavior relationships) versus the relative effect of PTSD effects on functional outcome (behavior-behavior relationships) is an ongoing area of research and debate, which some have proposed to be a distracting approach to the care of our veterans. [12]. Regardless, while adopting a dichotomous view may be clinically distracting, the literature needs to pursue the unique contributions of these comorbidities.

Future studies could address these limitations by including combat-related mild TBI and non-combat-related 
mild TBI comparison groups. Our study is limited by a small sample size that may have hampered our ability to detect stronger univariable psychological correlations with functional status, which is important considering all of our subjects had comorbid PTSD with or without additional depression. Likewise, our sample size was also prohibitively small to allow for typical multivariate modeling. Furthermore, because of the small sample size, we were not able to determine the best CLOX1 cutoff point score at which functional impairment emerges. This makes clinical interpretation of the CLOX1 difficult, especially given that the majority of subjects had normal CLOX1 scores. We had insufficient power to use multivariable modeling to control or adjust for the use of psychotropics or other potentially confounding medications. Also, our small sample size may have affected our correlation analyses since they can be influenced by outlier data points. However, we still detected significant differences in mean CLOX1 performance when we stratified the group into low- versus highperforming functional status groups. Finally, our neuropsychological measures consisted only of brief screening instruments. Although these are valid and reliable instruments, comprehensive neuro-psychological testing may have demonstrated other cognitive domains or other executive processes sensitive to post-TBI functional status. Additionally, while our chosen instruments are valid and reliable, we did not include a measure of effort, which could compromise our findings.

\section{CONCLUSIONS}

Regardless, we were able to demonstrate that an easily administered 5 min CLOX correlates well with functional status and discriminates between those with high versus low performance-based functional status scores. We know of no other studies that have examined the relationship between executive function and functional status following combat-related mild TBI in those with co-occurring PTSD. One unique aspect of our study was the use of performance-based functional measures that may be more objective than self- or proxy-reports. By identifying CLOX1 as a measure sensitive to performance-based functional status, we may be able to suggest practical, relevant screening tests to military physicians or other providers that could be used in the field and in clinical settings.

While neuropsychological and functional impairments are of tremendous concern to military personnel and civilians alike, the military combat environment pre- sents a unique set of operational functional requirements. Alterations in attention, flexibility and judgment, and impulse control could adversely affect combat functioning including driving, handling firearms, establishing situational awareness, following rules of engagement, and/or controlling aggression in conflict scenarios. Future studies should probe the possibility of using convenient but reliable clock drawing tasks to assist with both war zone return-to-duty decisions as well as with making more efficient referrals to neuropsychology for formal consultation in higher-echelon medical facilities. Moreover, these studies could further delineate neuropsychological targets for future intervention studies aimed at improving the cognitive deficits relevant to functional decline.

\section{ACKNOWLEDGMENTS}

\section{Author Contributions:}

Study concept and design: B. W. Writer, J. E. Schillerstrom. Acquisition of data: B. W. Writer, H. K. Regwan, B. S. Harlan. Analysis and interpretation of data: B. W. Writer, J. E. Schillerstrom. Drafting of manuscript: B. W. Writer, J. E. Schillerstrom,

H. K. Regwan, B. S. Harlan.

Critical revision of manuscript for important intellectual content:

B. W. Writer, J. E. Schillerstrom.

Statistical analysis: J. E. Schillerstrom.

Administrative, technical, or material support: B. W. Writer.

Study supervision: B. W. Writer, J. E. Schillerstrom.

Financial Disclosures: The authors have declared that no competing interests exist.

Funding/Support: This material was unfunded at the time of manuscript preparation.

Additional Contributions: The opinions expressed on this document are solely those of the authors and do not represent an endorsement by or the views of the U.S. Air Force, the DOD, or the U.S. Government. Institutional Review: We recruited all participants in accordance with the procedures approved by the joint institutional review boards (IRBs) of WHMC and Brooke Army Medical Center, Fort Sam Houston, Texas, as well as the University of Texas Health Science Center at San Antonio IRB and STVHCS IRB, Audie L. Murphy Division.

Participant Follow-Up: The authors do not plan to inform participants of the publication of this study.

\section{REFERENCES}

1. Hoge CW, McGurk D, Thomas JL, Cox AL, Engel CC, Castro CA. Mild traumatic brain injury in U.S. soldiers returning from Iraq. N Engl J Med. 2008;358(5):453-63.

[PMID: 18234750]

DOI:10.1056/NEJMoa072972 
2. Nampiaparampil DE. Prevalence of chronic pain after traumatic brain injury: A systematic review. JAMA. 2008; 300(6):711-19. [PMID: 18698069]

3. Warden D. Military TBI during the Iraq and Afghanistan wars. J Head Trauma Rehabil. 2006;21(5):398-402. [PMID: 16983225] DOI:10.1097/00001199-200609000-00004

4. The Management of Concussion/mTBI Working Group. VA/DoD clinical practice guideline for management of concussion/mild traumatic brain injury. J Rehabil Res Dev. 2009;46(6):CP1-68. [PMID: 20108447]

DOI:10.1682/JRRD.2009.06.0076

5. Kaufman DM. Clinical neurology for psychiatrists. 6th ed. Philadelphia (PA): Saunders; 2007. p. 537-48.

6. National Institute of Neurological Disorders, National Institutes of Health. Traumatic brain injury: Hope through research [Internet]. Washington (DC): National Institute of Neurological Disorders and Stroke; 2002 [cited 2008 Oct 20]. Available from: http://www.ninds.nih.gov/disorders/tbi/ tbi_htr.pdf.

7. Sadock BJ, Kaplan HI, Sadock VA. Kaplan \& Sadock’s synopsis of psychiatry: Behavioral sciences/clinical psychiatry. 10th ed. Philadelphia (PA): Wolter Kluwer/Lippincott Williams \& Wilkins; 2007. p. 363-64.

8. Stern TA, Rosenbaum JF, Fava M, Biederman J, Rauch SL. Massachusetts General Hospital comprehensive clinical psychiatry. Philadelphia (PA): Mosby/Elsevier; 2008. p. 1107-21.

9. Powell JM, Ferraro JV, Dikmen SS, Temkin NR, Bell KR. Accuracy of mild traumatic brain injury diagnosis. Arch Phys Med Rehabil. 2008;89(8):1550-55. [PMID: 18597735] DOI:10.1016/j.apmr.2007.12.035

10. Rao V, Lyketsos C. Neuropsychiatric sequelae of traumatic brain injury. Psychosomatics. 2000;41(2):95-103. [PMID: 10749946] DOI:10.1176/appi.psy.41.2.95

11. Erez AB, Rothschild E, Katz N, Tuchner M, HartmanMaeir A. Executive functioning, awareness, and participation in daily life after mild traumatic brain injury: A preliminary study. Am J Occup Ther. 2009;63(5):634-40.

[PMID: 19785263]

12. Hoge CW, Goldberg HM, Castro CA. Care of war veterans with mild traumatic brain injury-Flawed perspectives. N Engl J Med. 2009;360(16):1588-91. [PMID: 19369664] DOI:10.1056/NEJMp0810606

13. Rassovsky Y, Satz P, Alfano MS, Light RK, Zaucha K, McArthur DL, Hovda D. Functional outcome in TBI I: Neuropsychological, emotional, and behavioral mediators. J Clin Exp Neuropsychol. 2006;28(4):567-80. [PMID: 16624784]

DOI:10.1080/13803390500434466

14. Warden DL, Gordon B, McAllister TW, Silver JM, Barth JT, Bruns J, Drake A, Gentry T, Jagoda A, Katz DI, Kraus J, Labbate LA, Ryan LM, Sparling MB, Walters B, Whyte
J, Zapata A, Zitnay G; Neurobehavioral Guidelines Working Group. Guidelines for the pharmacologic treatment of neurobehavioral sequelae of traumatic brain injury. J Neurotrauma. 2006;23(10):1468-1501. [PMID: 17020483] DOI:10.1089/neu.2006.23.1468

15. Kersel DA, Marsh NV, Havill JH, Sleigh JW. Neuropsychological functioning during the year following severe traumatic brain injury. Brain Inj. 2001;15(4):283-96.

[PMID: 11299130] DOI:10.1080/02699050010005887

16. Pietrzak RH, Johnson DC, Goldstein MB, Malley JC, Southwick SM. Posttraumatic stress disorder mediates the relationship between mild traumatic brain injury and health and psychosocial functioning in veterans of Operations Enduring Freedom and Iraqi Freedom. J Nerv Ment Dis. 2009;197(10):748-53. [PMID: 19829203] DOI:10.1097/NMD.0b013e3181b97a75

17. Stein MB, McAllister TW. Exploring the convergence of posttraumatic stress disorder and mild traumatic brain injury. Am J Psychiatry. 2009;166(7):768-76.

[PMID: 19448186]

DOI:10.1176/appi.ajp.2009.08101604

18. Halbauer JD, Ashford JW, Zeitzer JM, Adamson MM, Lew HL, Yesavage JA. Neuropsychiatric diagnosis and management of chronic sequelae of war-related mild to moderate traumatic brain injury. J Rehabil Res Dev. 2009;46(6):757-96. [PMID: 20104402]

DOI:10.1682/JRRD.2008.08.0119

19. McDonald BC, Flashman LA, Saykin AJ. Executive dysfunction following traumatic brain injury: Neural substrates and treatment strategies. Neurorehabilitation. 2002;17(4): 333-44. [PMID: 12547981]

20. Niogi SN, Mukherjee P, Ghajar J, Johnson CE, Kolster R, Lee H, Suh M, Zimmerman RD, Manley GT, McCandliss BD. Structural dissociation of attentional control and memory in adults with and without mild traumatic brain injury. Brain. 2008;131(Pt 12):3209-21. [PMID: 18952679] DOI:10.1093/brain/awn247

21. Frencham KA, Fox AM, Maybery MT. Neuropsychological studies of mild traumatic brain injury: A meta-analytic review of research since 1995. J Clin Exp Neuropsychol. 2005;27(3):334-51. [PMID: 15969356] DOI:10.1080/13803390490520328

22. Nelson LA, Yoash-Gantz RE, Pickett TC, Campbell TA. Relationship between processing speed and executive functioning performance among OEF/OIF veterans: Implications for postdeployment rehabilitation. J Head Trauma Rehabil. 2009;24(1):32-40. [PMID: 19158594] DOI:10.1097/HTR.0b013e3181957016

23. Mathias JL, Coats JL. Emotional and cognitive sequelae to mild traumatic brain injury. J Clin Exp Neuropsychol. 1999;21(2):200-215. [PMID: 10425517]

DOI:10.1076/jcen.21.2.200.930 
24. Campbell TA, Nelson LA, Lumpkin R, Yoash-Gantz RE, Pickett TC, McCormick CL. Neuropsychological measures of processing speed and executive functioning in combat veterans with PTSD, TBI, and comorbid TBI/PTSD. Psychiatr Ann. 2009;39(8):796-803. DOI:10.3928/00485713-20090728-01

25. Raskin SA, Mateer CA, Tweeten R. Neuropsychological assessment of individuals with mild traumatic brain injury. Clin Neuropsychol. 1998;12(1):21-30.

26. Jefferson AL, Paul RH, Ozonoff A, Cohen RA. Evaluating elements of executive functioning as predictors of instrumental activities of daily living (IADLs). Arch Clin Neuropsychol. 2006;21(4):311-20. [PMID: 16814980] DOI:10.1016/j.acn.2006.03.007

27. Royall DR, Lauterbach EC, Kaufer D, Malloy P, Coburn KL, Black KJ; Committee on Research of the American Neuropsychiatric Association. The cognitive correlates of functional status: A review from the Committee on Research of the American Neuropsychiatric Association. J Neuropsychiatry Clin Neurosci. 2007;19(3):249-65.

[PMID: 17827410]

DOI:10.1176/appi.neuropsych.19.3.249

28. Rassovsky Y, Satz P, Alfano MS, Light RK, Zaucha K, McArthur DL, Hovda D. Functional outcome in TBI II: Verbal memory and information processing speed mediators. J Clin Exp Neuropsychol. 2006;28(4):581-91. [PMID: 16624785] DOI:10.1080/13803390500434474

29. Royall DR, Cordes JA, Polk M. CLOX: An executive clock drawing task. J Neurol Neurosurg Psychiatry. 1998; 64(5):588-94. [PMID: 9598672]

DOI:10.1136/jnnp.64.5.588

30. Royall DR, Mulroy AR, Chiodo LK, Polk MJ. Clock drawing is sensitive to executive control: A comparison of six methods. J Gerontol B Psychol Sci Soc Sci. 1999;54(5): P328-33. [PMID: 10542825]

31. Lavery LL, Starenchak SM, Flynn WB, Stoeff MA, Schaffner R, Newman AB. The clock drawing test is an independent predictor of incident use of 24-hour care in a retirement community. J Gerontol A Biol Sci Med Sci. 2005;60(7):928-32. [PMID: 16079220]

32. Folstein MF, Folstein SE, McHugh PR. "Mini-Mental State.” A practical method for grading the cognitive state of patients for the clinician. J Psychiatr Res. 1975;12(3):189-98. [PMID: 1202204$]$

DOI:10.1016/0022-3956(75)90026-6

33. Royall DR, Mahurin RK, Gray KF. Bedside assessment of executive cognitive impairment: The executive interview. J Am Geriatr Soc. 1992;40(12):1221-26.

[PMID: 1447438]

34. Buschke H, Kuslansky G, Katz M, Stewart WF, Sliwinski MJ, Eckholdt HM, Lipton RB. Screening for dementia with the memory impairment screen. Neurology. 1999;52(2): 231-38. [PMID: 9932936]

35. Weathers FW, Litz BT, Herman DS, Huska JA, Keane TM. The PTSD Checklist (PCL): Reliability, validity, and diagnostic utility. Annual Meeting of the International Society for Traumatic Stress Studies; 1993 Oct; San Antonio, TX.

36. Hamilton M. A rating scale for depression. J Neurol Neurosurg Psychiatry. 1960;23:56-62. [PMID: 14399272] DOI:10.1136/jnnp.23.1.56

37. San Antonio Longitudinal Study of Aging (SALSA). Manual of operational definitions for variables in the SALSA Disablement Process Model. San Antonio (TX): The University of Texas Health Science Center; 1999.

38. Keen SM, Kutter CJ, Niles BL, Krinsley KE. Psychometric properties of PTSD Checklist in sample of male veterans. J Rehabil Res Dev. 2008;45(3):465-74. [PMID: 18629754] DOI:10.1682/JRRD.2007.09.0138

Submitted for publication October 5, 2009. Accepted in revised form June 9, 2010.

This article and any supplementary material should be cited as follows:

Writer BW, Schillerstrom JE, Regwan HK, Harlan BS. Executive clock drawing correlates with performancebased functional status in people with combat-related mild traumatic brain injury and comorbid posttraumatic stress disorder. J Rehabil Res Dev. 2010;47(9):841-50.

DOI:10.1682/JRRD.2009.10.0162

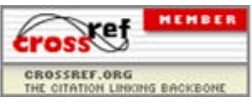


